\title{
Goals, organizational change, advocacy, diversity literacy, and sustainability: A checklist for diversity in cardiothoracic surgery training programs
}

\author{
Katherine A. Ortmeyer, BA, ${ }^{\mathrm{a}}$ Vignesh Raman, MD, ${ }^{\mathrm{b}}$ Chidinma S. Tiko-Okoye, MD, MPH, \\ Jairo A. Espinosa, MD, ${ }^{\mathrm{d}}$ David T. Cooke, MD, ${ }^{\mathrm{e}}$ Sandra L. Starnes, MD, ${ }^{\mathrm{f}}$ and Cherie P. Erkmen, $\mathrm{MD}^{\mathrm{d}}$
}

Diversity in a physician workforce is essential to effectively treat a rapidly changing patient population and optimize the modern healthcare environment. Physicians must be culturally competent and integrate a diversity of language, communication styles, beliefs, and attitudes into patient care. ${ }^{1}$ Delivery of equitable health care depends on a diverse physician workforce, which has been proven to result in improved patient satisfaction and quality of care, ${ }^{2}$ as well as superior financial performance for health systems. ${ }^{3}$ Outside of health care, diversity leads to improved company culture, talent retention, and increased innovation. ${ }^{4}$ Innovative solutions and adaptability are essential in the face of major shifts in healthcare paradigms, such as those unfolding during the Coronavirus Disease 2019 pandemic, and will absolutely require a diverse physician workforce.

Despite evidence in favor of a diverse workforce, diversity among physicians remains low, particularly in surgical subspecialties. Among academic cardiothoracic (CT) surgeons in 2018, Black and Hispanic surgeons composed only $3 \%$ and $5 \%$ of the workforce, respectively, despite representing $13 \%$ and $18 \%$ of the general US population. ${ }^{5}$ Women represented only $17 \%$ of practicing CT surgeons. Representation of these groups is even lower among senior and leadership positions. ${ }^{6}$ Furthermore, trainees and surgeons have reported high rates of racial discrimination,

From the ${ }^{\text {a Lewis }}$ Katz School of Medicine at Temple University, Philadelphia, Pa; ${ }^{\mathrm{b}}$ General Surgery Residency Program, Duke University School of Medicine, Durham, NC; ${ }^{\mathrm{c}}$ Department of Surgery, Center for Population Health, Lankenau Medical Center, Wynnewood, Pa; ${ }^{\mathrm{d} D e p a r t m e n t ~ o f ~ T h o r a c i c ~ M e d i c i n e ~ a n d ~ S u r g e r y, ~}$ Lewis Katz School of Medicine at Temple University, Philadelphia, Pa; ${ }^{\text {eSection of }}$ General Thoracic Surgery, UC Davis Health, Sacramento, Calif; and ${ }^{\mathrm{f}}$ Division of Thoracic Surgery, University of Cincinnati, Cincinnati, Ohio.

This study was partially supported by TUFCCC/HC Regional Comprehensive Cancer Health Disparity Partnership, Award Number U54 CA221704(5) (Contact Principal Investigators: Grace X. Ma, PhD, and Olorunseun O. Ogunwobi, MD, $\mathrm{PhD}$ ) from the National Cancer Institute of National Institutes of Health. The content is solely the responsibility of the authors and does not necessarily represent the official views of the National Cancer Institute of National Institutes of Health.

Received for publication Aug 13, 2020; revisions received Oct 18, 2020; accepted for publication Nov 14, 2020; available ahead of print Dec 3, 2020.

Address for reprints: Cherie P. Erkmen, MD, Department of Thoracic Medicine and Surgery, Lewis Katz School of Medicine, Temple University, Parkinson Pavilion, Zone C, Suite 501, 3401 N Broad St, Philadelphia, PA 19140 (E-mail: Cherie. erkmen@tuhs.temple.edu).

J Thorac Cardiovasc Surg 2021;162:1782-7

$0022-5223 / \$ 36.00$

Copyright (c) 2020 by The American Association for Thoracic Surgery

https://doi.org/10.1016/j.jtcvs.2020.11.112

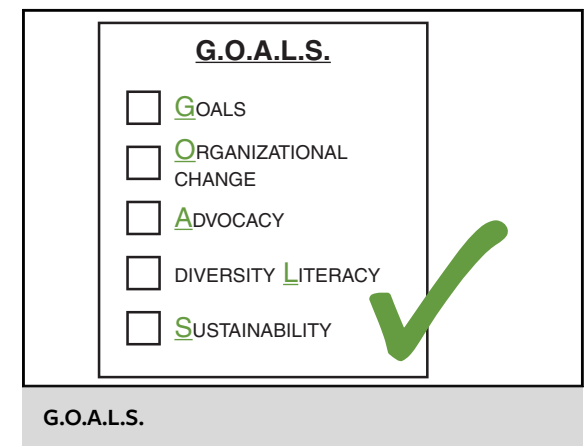

CENTRAL MESSAGE

In our G.O.A.L.S. checklist, setting goals, organizational change, advocacy, diversity literacy, and sustainability are key for promoting D\&l in CT surgery programs.

See Commentaries on pages 1788 and 1789.

gender discrimination, and harassment that adversely affect physician wellness. ${ }^{7,8}$ The President Emeritus of the Association of American Medical Colleges (AAMC) addressed this subject in 2019, writing "now more than ever, we must take decisive action to end conscious and unconscious racial bias, overt discrimination, gender harassment and inequity in academic medicine." 9 The Accreditation Council for Graduate Medical Education (ACGME) has also recognized the importance of developing a culture of diversity by adding to the common program requirements: "[Each program] must engage in practices that focus on mission-driven, ongoing, systematic recruitment and retention of a diverse and inclusive workforce of residents, fellows, faculty members, senior administrative staff members, and other relevant members of its academic community." 10 Unfortunately, there are few guidelines for training programs regarding implementation of a culture of diversity and inclusion (D\&I). To address this, we propose a checklist to serve as a comprehensive framework 
for the development of a program-specific D\&I policy within every CT surgery program (Figure 1). This is a necessary first step, and as more evidence is gathered on the efficacy of initiatives, more directive best practices may be developed in the future.

\section{MATERIALS AND METHODS}

A MEDLINE and PubMed search was conducted using the terms "diversity," "inclusion," "surgery," and "thoracic surgery." Guidelines and evidence were gathered from the ACGME, AAMC, the American College of Surgeons, the American Surgical Association, various university surgical programs, and publications in psychology, sociology, and business. Recommendations were developed on the basis of a review of these resources.

\section{DISCUSSION}

Our search returned hundreds of articles on D\&I in surgery and medicine and thousands more in fields outside of health care. Quantities increased every year, revealing an increased awareness of the importance of diversity. We focused on several key guidelines, articles, and studies in developing our recommendations.

\section{Themes}

Five themes emerged as critical to the development of a successful D\&I program: (1) the establishment of discrete and specific goals, (2) organizational change to support initiatives, (3) $\boldsymbol{a}$ dvocacy and support structures to attract and retain diverse surgeons, (4) educational programs for diversity $l$ iteracy and culture change, and (5) sustainability of efforts. Think Goals, Organizational change, Advocacy, Literacy, Sustainability (G.O.A.L.S.) (Figure 2).

\section{Goals}

The first critical component of a diversity program is the establishment of discrete goals. ${ }^{11}$ An initial step is the creation of a mission statement that defines the program's commitment to diversity, followed by a vision statement of where the organization sees itself after a period of sustained effort. Programs may revise their overall mission statement or create a separate mission statement regarding D\&I. Each program should define its own goals for diversity and systematic processes, or deliverables, to evaluate accomplishments or failures. ${ }^{11}$ Potential metrics of progress include the number of applicants, interviewees, and trainees who are women or under-represented minorities in medicine (UIM). Training programs should also establish goals for recruitment, retention, and promotion of faculty from underrepresented populations. Advancement of women and UIM faculty not only improves faculty diversity but also increases recruitment of these same groups by improving applicants' and trainees' perception of opportunity in the field. ${ }^{12}$ At the faculty level, systems of compensation, evaluation, and promotion must be scrutinized for bias, sexism, and racism by members of the D\&I task force through collaborative roundtable forums or with the assistance of a third party. ${ }^{13}$ The diversity mission, goals, and policy should be disseminated to faculty and trainees, as well as potential applicants through recruitment materials and online program descriptions including the AAMC and ACGME websites. Programs should aim to produce scholarship related to the performance of its diversity efforts. These experiences should be shared in national meetings

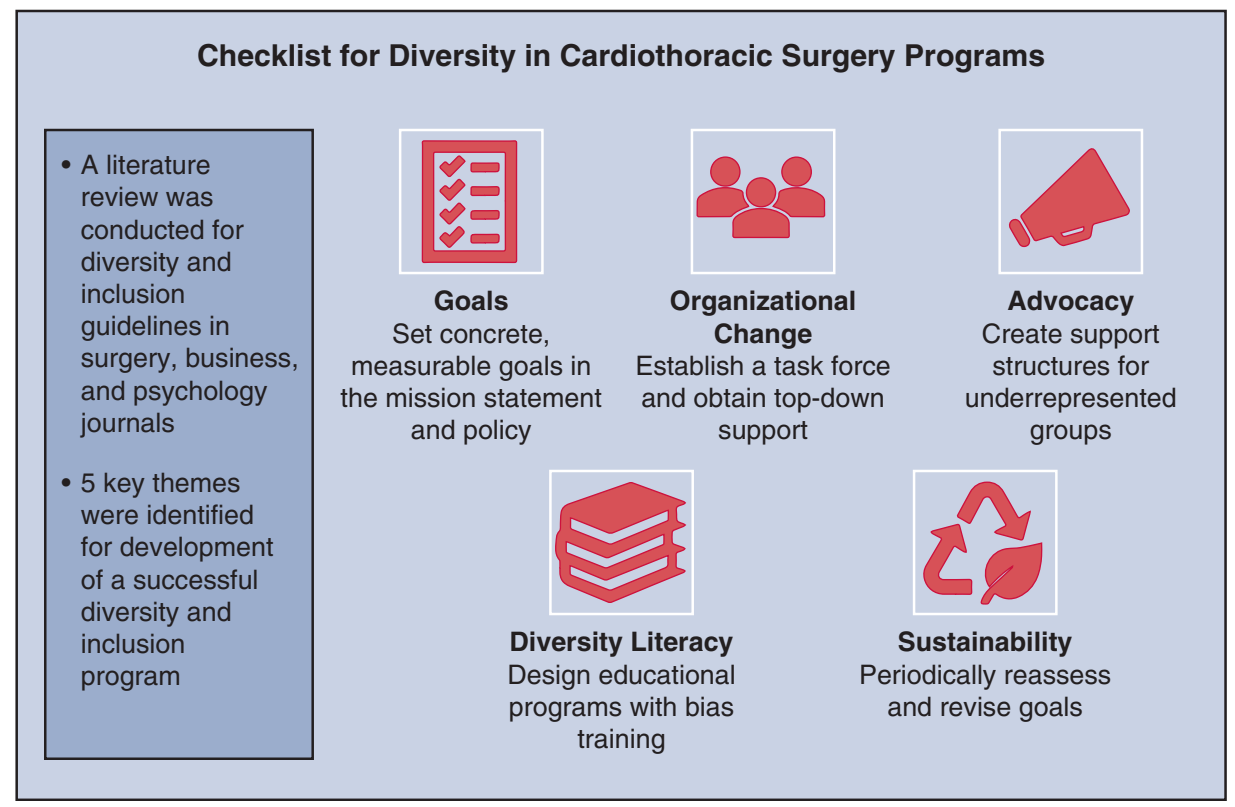

FIGURE 1. G.O.A.L.S. 
Establish discrete and actionable goals for the program

- Develop a mission statement and policy specific to the program

- Collect data on existing disparities

- Set concrete and attainable goals for improving diversity

- Establish metrics, monitor program progress

Obtain top-down support and promote organizational change

- Establish a taskforce for development of and adherence to the diversity policy

- Empower taskforce with recognition/compensation/administrative support

- Include taskforce members on committees involving salaries \& promotion

- Collaborate with existing diversity officers in the health system or academic center

$\square$ Create advocacy and support structures for under-represented groups

- Create summer internships/shadowing opportunities for students and residents

- Engage in active recruitment with local and national organizations

- Establish formal mentorship programs for underrepresented groups

- Train mentors to recognize discrimination, harassment and bias

- Create safe spaces and support groups for underrepresented groups

Increase resident and faculty diversity literacy through educational programs

- Emphasize importance of diversity in healthcare providers

- Define adverse effects of discrimination, microaggression and implicit bias

- Implement implicit bias training

- Define mechanisms of reporting discrimination, protection from retaliation and accountability measures for instances of harassment and abuse

- Use inclusive language in departmental and section communications and advertising

- Publish on performance of diversity efforts

$\square$ Plan for sustainability and continued efforts

- Reassess goals periodically and revise based on experience

FIGURE 2. Checklist for diversity and inclusion in CT surgery.

and peer-reviewed publications to disseminate successful strategies to mitigate structural bias in CT surgery.

\section{Organizational change}

Efforts to promote diversity in a CT surgery training program require organizational support and depend on buy-in from institutional leadership, administrators, faculty, trainees, and support staff. ${ }^{14}$ Each program should establish a task force or responsible parties to uphold the mission. ${ }^{15}$ The task force must seek to understand the barriers to diversity within the program, prioritize areas that can be most effectively targeted for change, ${ }^{16}$ and develop actionable goals. ${ }^{17}$ A number of tools are available to assist in this process, including the Diversity Engagement Survey ${ }^{18}$ and the National Institutes of Health Scientific Workforce Diversity Toolkit. ${ }^{19}$ It is also the responsibility of the task force to assess current institutional resources and identify additional investments needed to achieve diversity goals. ${ }^{16}$

Sponsoring institutions of training programs must actively empower the diversity task force to achieve diversity goals. Leadership of departments, health systems, and medical schools can validate the task force with participation from leadership, recognition, resources, administrative personnel, and ideally compensation for invested effort. ${ }^{14,20}$ Faculty who contribute to diversity efforts in meaningful ways such as mentoring UIM trainees, developing diversity curricula, or reorganizing recruitment to mitigate bias could receive compensation for these actions. Forms of compensation may include a reduction in clinical workload, credit through an Educational Value Unit in a relative value unit-based performance system or acknowledgement of these valued endeavors during promotions. ${ }^{21}$ 
Finally, programs should collaborate with existing D\&I officers, representatives or experts within institutions who can assist in optimizing D\&I in processes of recruitment, retention, evaluation, and promotion of trainees and faculty. Diversity specialists within the sponsoring institution can align training program goals and create shared resources. For example, some institutions sponsor housing or transportation for UIM students and trainees to interview or attend a second-look visit to any residency in their system. ${ }^{12}$ Other potential strategies that can be shared among training programs within an institution include the development of websites and recruitment materials that highlight the diversity mission and policies of the institution ${ }^{22}$ and outreach and mentorship programs to attract underrepresented populations such as pipeline programs, ${ }^{23}$ "surgical shadowing schemes," ${ }^{24}$ or funded sub-internship opportunities. ${ }^{25}$ Some training programs have successfully used outside consultants to engage faculty and trainees in sensitive and sometimes emotional discussions about bias and discrimination. Like all diversity efforts, for this approach to be successful, program and institutional leadership must dedicate money, time and effort.

\section{Advocacy}

To attract and retain women and UIM surgeons in a CT surgery program, it is important to provide a formal support system to advocate for them. The decision to pursue a specialty is often made early in undergraduate training or medical school. Therefore, CT surgery programs should strive to improve the exposure of UIM individuals to the specialty through summer research internships for students, clinical shadowing opportunities, and collaboration with student organizations such as the Latino Medical Student Association, Society of Black Academic Surgeons, Student National Medical Association and Association of Women Surgeons. Training programs should actively encourage women and UIM students to apply for scholarships offered by CT surgery societies and to attend national CT surgery meetings. Because UIM individuals are less likely to receive mentorship, formal mentoring programs should be developed to support these individuals throughout training. ${ }^{17}$ Ideally, programs can develop a network of mentorship among students, residents, fellows, and faculty at different stages of their career. These relationships are fundamental to establishing a culture of inclusion. ${ }^{17} \mathrm{Men}-$ tors should be educated and prepared to recognize discrimination, harassment, and bias through grand-rounds lectures or small group seminars and should be able to refer mentees to appropriate resources for help. ${ }^{26}$ Among the care team, mentors and faculty should coach all trainees in promoting inclusion and respect. The responsibility for mentorship should not fall disproportionately on women and underrepresented minority surgeons in the program. ${ }^{27}$ Every surgeon should be encouraged to mentor people of differing backgrounds, UIM individuals, and women. ${ }^{28}$

Once an UIM or female faculty member is recruited, they continue to require advocacy and support from the institution, particularly through access to professional development opportunities. These opportunities include but are not limited to grant writing workshops, time management didactics, personal finance education, administrative assistant support, realistic performance expectations in the tripartite academic mission, and transparent criteria for promotion and merit advancement. Finally, we must acknowledge the profound loneliness that can burden the scarce diverse faculty working in our field. With Black CT surgeons composing only $3 \%$ of academic CT surgery faculty, statistically each Black surgeon is likely the only Black academic in the room. This can have overwhelming effects on one's psyche. This potential loneliness must be acknowledged by institutional leadership, and support groups and safe spaces, whether in person or through technological conferencing, should be provided. ${ }^{29}$

\section{Literacy}

A critical component of a diversity policy is the development of educational programs to support a culture of diversity and mitigate structural bias. ${ }^{30}$ Programs can reference several online resources ${ }^{19,31}$ or consult their own institutional diversity offices for assistance in developing a diversity curriculum. To start, programs can share evidencebased data regarding the importance of supporting diversity. Faculty and trainees should understand that discrimination based on race, gender, national origin, or sexual orientation violates the Civil Rights Act of 1964. Subtle discrimination and bias cannot be tolerated according to the program mission policy. Faculty and trainees should also receive current evidence on the prevalence of discrimination and bias within the field of CT surgery to highlight the urgency for action. Faculty should receive education on mitigating bias, especially in the setting of recruitment, ${ }^{32}$ but also in evaluation and promotion of trainees as well as faculty, as specified in the ACGME Common Program Requirements.

Programs should develop a curriculum that includes what to do if a trainee or faculty member is experiencing or witnessing discrimination. Academic institutions usually have an Office of Equal Employment Opportunity to guide individuals and programs through these challenging events. Alternatively, hospital systems may have a separate mechanism of reporting through Offices of Human Resources or through section or divisional leadership. Regardless, the process of reporting should be clear to all trainees and faculty. Additionally, everyone must understand that retaliation or ostracism as a result of reporting discrimination or bias is unlawful. Education should address "microaggressions" or less egregious but widespread and harmful actions 
rooted in bias. In CT surgery, these include belittling comments, suggesting that women or minorities did not earn their position in CT surgery, assuming that women or minorities are not CT fellows, and excluding women or minorities from discussions, rounds, or social gatherings. Ignoring or minimizing microaggressions supports structural bias, and stakeholders may come to the erroneous conclusion that they must tolerate microaggression as part of CT surgery culture. Therefore, microaggressions must be definitively acknowledged and addressed to create a safer and more equitable environment. Additionally, faculty and trainees should understand and assess their own implicit biases with tools like the Harvard Implicit Association Test. ${ }^{33}$ Microaggressions and implicit bias can be more damaging to an academic culture than overt discrimination because they are ubiquitous and more likely to be tolerated. ${ }^{34}$ Each program should develop a policy for addressing microaggression and implicit bias. As part of the educational curriculum, the mission, goals and policies developed to support diversity should be regularly updated and disseminated among faculty, trainees, and staff.

\section{Sustainability}

ACGME has newly highlighted diversity in 2019 and world events of Coronavirus Disease 2019 and national protests regarding racial injustice in 2020 have compelled an introspection regarding structural racism. Although we propose a checklist to promote $D \& I$ in a training program, this is not a task that can be checked off and forgotten. D\&I efforts must be sustained and integrated into everyday practice. Achievement of diversity goals, whether they relate to recruitment, mentoring, or education, must be assessed at least annually and revised based on experience. If programs are not accomplishing diversity goals, this should prompt a needs assessment-rather than doubling down on potentially misguided initiatives, reevaluation should occur to ensure that areas of improvement are being targeted. Framing diversity initiatives in a positive light, rather than with negative or punitive language, is also key for sustainability. ${ }^{16}$ Training programs and sponsoring institutions must support and compensate individuals demonstrating commitment to diversity efforts. Finally, faculty and trainees should look for innovative opportunities within their program and nationally to support a diverse workforce and an inclusive environment.

\section{Study Limitations}

There are limitations to this checklist. We acknowledge that galvanizing commitment to D\&I is easier said than done. Our recommendations cannot be realistically applied without significant buy-in at all levels including trainees, faculty, chiefs, chairs, and most important, health system leadership. Second, our checklist does not address the significant time and financial resources necessary for an effective D\&I program. The formation of a diversity task force and the implementation of outreach and "in-reach" efforts require significant time, which even interested CT surgery faculty may not be able to provide. These constraints should be candidly acknowledged as each CT surgery program develops realistic goals for diversity. Finally, the recommendations listed here represent consensus themes from literature drawn from numerous academic fields and are not tailored to the needs of individual CT surgery programs. Because programs are likely at different levels of adherence to the ACGME requirement for a diverse and inclusive workforce, we propose this checklist as a starting point. Further studies into effective strategies among CT surgery training programs are needed. Hopefully, with collaboration of all CT surgery training programs, we can develop best practices for implementing a culture of D\&I and dismantling structural racism.

\section{CONCLUSIONS}

Addressing structural racism and improving D\&I have a positive impact on physician well-being and patient care. Diversity is essential to the vitality and growth of CT surgery, because if we do not draw from the broader population, we will not attract the best and brightest. In this work, we present a G.O.A.L.S. Checklist framework for the creation of a D\&I policy within a CT surgery program. This checklist is guided by themes of establishing actionable goals, organizational change, longitudinal advocacy, diversity literacy, and sustainability. These principles can be used as a template for CT surgery programs seeking to achieve a diverse and inclusive training environment.

\section{Conflict of Interest Statement}

The authors reported no conflicts of interest.

The Journal policy requires editors and reviewers to disclose conflicts of interest and to decline handling or reviewing manuscripts for which they may have a conflict of interest. The editors and reviewers of this article have no conflicts of interest.

\section{References}

1. Paez KA, Allen JK, Beach MC, Carson KA, Cooper LA. Physician cultural competence and patient ratings of the patient-physician relationship. J Gen Intern Med. 2009;24:495-8.

2. Saha S, Komaromy M, Koepsell TD, Bindman AB. Patient-physician racial concordance and the perceived quality and use of health care. Arch Intern Med. 1999; 159:997-1004.

3. Gomez LE, Bernet P. Diversity improves performance and outcomes. J Natl Med Assoc. 2019;111:383-92

4. The 2019/2020 Hays Asia diversity \& inclusion report. Available at: http://image. email.hays.com/lib/fe4315707564057c751c71/m/1/f344ca83-da48-4fe5-9fb9f4c02252159a.pdf. Accessed October 17, 2020.

5. Ortmeyer K, Raman V, Tiko-Okoye C, Espinosa J, Cooke DT, Erkmen C. Women and minorities underrepresented in academic cardiothoracic surgery: it's time for next steps. Ann Thorac Surg. November 5, 2020 [Epub ahead of print]. 
6. Cooke DT, Olive J, Godoy L, Preventza O, Mathisen DJ, Prager RL. The importance of a diverse specialty: introducing the STS workforce on diversity and inclusion. Ann Thorac Surg. 2019;108:1000-5.

7. Hu Y-Y, Ellis RJ, Hewitt DB, Yang AD, Cheung EO, Moskowitz JT, et al. Discrimination, abuse, harassment, and burnout in surgical residency training. N Engl J Med. 2019;381:1741-52.

8. Filut A, Alvarez M, Carnes M. Discrimination toward physicians of color: a systematic review. J Natl Med Assoc. 2020;112:117-40.

9. Addressing racism and mistreatment in academic medicine. AAMC. Available at: https:/www.aamc.org/news-insights/addressing-racism-and-mistreatment-ac ademic-medicine. Accessed July 22, 2020.

10. Common program requirements. Available at: https://www.acgme.org/What-WeDo/Accreditation/Common-Program-Requirements. Accessed May 26, 2020.

11. Kaiser CR, Major B, Jurcevic I, Dover TL, Brady LM, Shapiro JR. Presumed fair: ironic effects of organizational diversity structures. J Pers Soc Psychol. 2013; 104:504-19.

12. Auseon AJ, Kolibash AJ, Capers Q. Successful efforts to increase diversity in a cardiology fellowship training program. J Grad Med Educ. 2013;5:481-5.

13. DiBrito SR, Lopez CM, Jones C, Mathur A. Reducing implicit bias: association of women surgeons \#heforshe task force best practice recommendations. J Am Coll Surg. 2019;228:303-9.

14. Kang SK, Kaplan S. Working toward gender diversity and inclusion in medicine: myths and solutions. Lancet. 2019:393:579-86.

15. Kalev A, Dobbin F, Kelly E. Best practices or best guesses? assessing the efficacy of corporate affirmative action and diversity policies. Am Sociol Rev. 2006;71: 589-617.

16. Pedulla D. Diversity and inclusion efforts that really work. Harvard business review. Available at: https://hbr.org/2020/05/diversity-and-inclusion-efforts-thatreally-work. Accessed May 18, 2020

17. Daley SP, Palermo A-G, Nivet M, Soto-Greene ML, Taylor VS, Butts GC, et al. Diversity in academic medicine no. 6 successful programs in minority faculty development: ingredients of success. Mt Sinai J Med. 2008; $75: 533-51$.

18. Diversity engagement Survey. AAMC. Available at: https://www.aamc.org/whatwe-do/mission-areas/diversity-inclusion/engagement-survey. Accessed October 17,2020 .

19. NIH scientific workforce diversity Toolkit | SWD at NIH. Available at: https:// diversity.nih.gov/toolkit. Accessed October 16, 2020.

20. Taggar S, Ellis R. The role of leaders in shaping formal team norms. Leadersh $Q$. 2007; 18:105-20.
21. Values, RVUs and teaching. International Association of Medical Science Educators - IAMSE. Available at: http://www.iamse.org/mse-article/values-rvusand-teaching/. Accessed July 22, 2020.

22. Kerr H-L, Armstrong LA, Cade JE. Barriers to becoming a female surgeon and the influence of female surgical role models. Postgrad Med J. 2016;92:576-80.

23. Mason BS, Ross W, Chambers MC, Grant R, Parks M. Pipeline program recruits and retains women and underrepresented minorities in procedure based specialties: a brief report. Am J Surg. 2017;213:662-5.

24. Bartlett RD, Momin SM, Azam S, Rotimi O, Quick TJ. Development of a "Surgical Shadowing Scheme" to improve undergraduate experiences of surgery. Adv Med Educ Pract. 2019;10:619-26.

25. Diversity sub internships | Center for health equity, diversity \& inclusion. Available at: https://cedi-web01.s.uw.edu/residents-fellows/diversity-visiting-medicalstudent-subinternship-program/. Accessed October 17, 2020.

26. Pendleton AA, Coe MTM, Saillant NN. How to define and address gender discrimination and sexual harassment in surgical training. American College of Surgeons. Available at: https://www.facs.org/education/division-of-education/ publications/rise/articles/gender. Accessed October 16, 2020.

27. Rodríguez JE, Campbell KM, Pololi LH. Addressing disparities in academic medicine: what of the minority tax? BMC Med Educ. 2015;15:6.

28. Byerley JS. Mentoring in the era of \#MeToo. JAMA. 2020;323:1714-5.

29. Gewin V. What Black scientists want from colleagues and their institutions Nature. 2020;583:319-22.

30. Devine PG, Forscher PS, Austin AJ, Cox WTL. Long-term reduction in implici race bias: prejudice habit-breaking intervention. J Exp Soc Psychol. 2012;48: 1267-78.

31. Diversity and inclusion toolkit resources. AAMC. Available at: https://www.aa mc.org/professional-development/affinity-groups/cfas/diversity-inclusion-toolkit/ resources. Accessed July 23, 2020.

32. Erkmen CP, Kane L, Cooke DT. Bias Mitigation in Cardiothoracic Recruitment Ann Thorac Surg. Available at: https://www.ncbi.nlm.nih.gov/pmc/articles/PM C7368657/. Accessed July 28, 2020.

33. Take a test. Available at: https://implicit.harvard.edu/implicit/takeatest.html. Accessed July 23, 2020

34. King E, Jones K. Why subtle bias is so often worse than blatant discrimination. Harvard business review. Available at: https://hbr.org/2016/07/why-subtle-biasis-so-often-worse-than-blatant-discrimination. Accessed May 22, 2020.

Key Words: checklist, diversity, inclusion 